\title{
Organic molecular crystal-based photosynaptic devices for an artificial visual-perception system
}

\author{
Wei Deng $\mathbb{D}^{1}$, Xiujuan Zhang', Ruofei Jia', Liming Huang' ${ }^{1}$ Xiaohong Zhang ${ }^{1}$ and Jiansheng Jie $\mathbb{B}^{1}$
}

\begin{abstract}
Recreating the visual-perception properties using organic electronic devices is highly desired for visual prosthetics and artificial intelligence. Although the integration of organic light-sensing components with synaptic devices can realize the recognition and memory functions for perceived images, complicated problems in device integration for practical applications are generally encountered. Here we demonstrate a new type of organic photosynaptic device based on organic molecular crystals, which can provide optical-sensing and synaptic functions together in one device by means of a unique photon-induced charge transfer effect. This device successfully emulates the working principles of human visual perception in terms of short-term plasticity, long-term potentiation, and spike-timing-dependent plasticity.

Moreover, a proof-of-concept artificial image-perception system is demonstrated by integrating the photosynapses on a flexible substrate. The new devices using organic semiconductors may open up innovative application areas, such as artificially intelligent electronic and perception systems, and facilitate the integration of such devices into nextgeneration flexible and stretchable electronics.
\end{abstract}

\section{Introduction}

Human visual perception refers to the brain's ability to decipher what the eyes see, which corresponds to a remarkable imaging and memory system ${ }^{1-3}$. Figure 1 illustrates the visual-perception process in the brain. The eyes receive incoming light from the external environment and focus it onto the retina, where an image of the visual stimulus is captured. Nerve cells in the retina as photoreceptors can convert visual information (light) into electrical impulses, which can travel along the optic nerve to the visual cortex at the back of the brain. The visual cortex contains a very large number of neurons. Then the neuronal network will arrange the features of the visual information in a meaningful way. Finally, the neuronal network will interpret the visual information so that we can perceive what we see. In the neural network, synapses act as a basic unit to transmit, memorize, recognize, and learn the visual information ${ }^{4-7}$. Visual perception is the

Correspondence: Jiansheng Jie (jsjie@suda.edu.cn)

${ }^{1}$ Institute of Functional Nano \& Soft Materials (FUNSOM), Jiangsu Key Laboratory for Carbon-Based Functional Materials \& Devices, Soochow University, Suzhou, Jiangsu 215123, P. R. China main channel for humans to obtain information. Recreation of the visual-perception properties using electronic devices could have profound implications for visual prosthetics and artificial intelligence ${ }^{8}$. For example, an artificial visual-perception system could help blind people regain their visual senses. In light of the theories of and observations on visual perception, an artificial visualperception device requires multifunctional integration of a light-sensing device similar to the retina and a signalmanagement system similar to the brain.

Organic semiconducting materials with unique features of long-term biocompatibility, good mechanical flexibility, and molecular diversity are ideal candidates for bionic perception devices ${ }^{9-11}$. Recent research efforts on artificial visual-perception systems have achieved a high level of sophistication using organic electronic materials ${ }^{12-14}$. Mostly, organic light-sensing components and synaptic devices were integrated into these systems to realize the recognition and memory functions for perceived signals. For example, Liu and co-workers reported the integration of organic heterojunction photodetectors and organic transistors with a ferroelectric dielectric as an artificial 


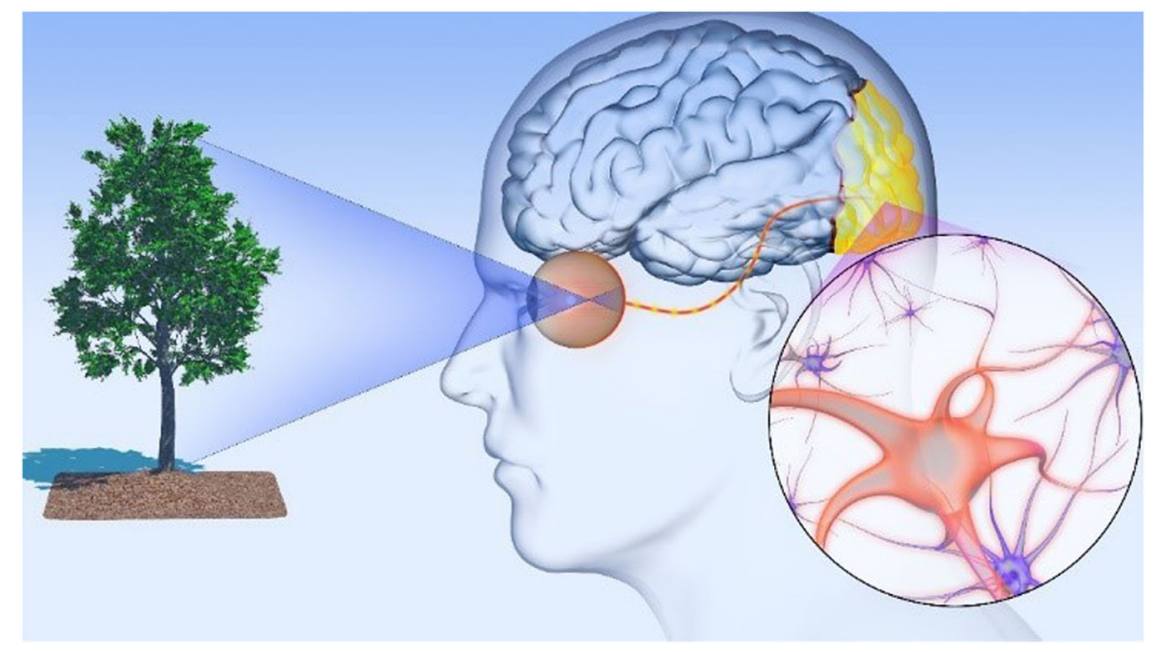

Fig. 1 Schematic illustration of the human visual-perception process.

visual-perception system ${ }^{12}$. Lee et al. also demonstrated an optic-neural synaptic device by integrating an organic photovoltaic detector with an organic nanowire synaptic transistor ${ }^{13}$. These systems successfully achieved light information processing and readout with synaptic plasticity, but they generally face complicated device integration problems for practical applications. In addition, these photodetectors made from polycrystalline films with many defects and grain boundaries exhibited small photoconduction changes under weak light illumination, leading to ambiguous signals with a low signal-to-noise ratio $^{15}$. Compared with polycrystalline thin films, organic molecular crystals (OMCs) with fewer defects and grain boundaries can dramatically enhance charge transport ${ }^{16-19}$, thereby enabling a large stimulated response in OMC-based neuromorphic devices. Therefore, implementing optical-sensing and synapsis functions together in one device based on OMCs is expected to provide a new and simple approach to artificial visual-perception systems.

Herein we demonstrate OMC-based photosynapses using a single device. The high crystal quality of OMCs ensures the fabrication of high-sensitivity photodetectors with a photoresponsivity up to $1650 \mathrm{~A} \mathrm{~W}^{-1}$ at a low gate voltage of $5 \mathrm{~V}$. In addition, photon-induced charge transfer from the OMCs to oxygen-induced deep traps occurs under light irradiation, enabling the storage of photogenerated holes to achieve the functions of synapses. Based on these unique properties, we have realized various functions of biological neural systems for visual perception in organic photosynaptic devices, including the three important forms of short-term plasticity (STP), long-term potentiation (LTP), and spike-timingdependent plasticity (STDP). Furthermore, a proof-ofconcept artificial image-perception sensor is constructed on a flexible substrate, possessing the capability to recognize and remember optical images. Given their solution-processing capability, outstanding device characteristics, and high device flexibility, organic photosynaptic devices present unique opportunities for future artificially intelligent electronic and perception systems.

\section{Results and discussion}

In this study, 5,11-bis(triethylsilylethynyl) anthradithiophene (Dif-TES-ADT) crystal arrays were used as photoactive layers due to their broad light absorption spectrum $(300-650 \mathrm{~nm})$, excellent air stability, and high carrier mobility $\left(\sim 6 \mathrm{~cm}^{2} \mathrm{~V}^{-1} \mathrm{~s}^{-1}\right)^{20-23}$. Details of the fabrication and characterization of Dif-TES-ADT crystal arrays can be found in Supplementary Figs. S1 and S2. The high carrier mobility of the organic crystals can accelerate the photocarrier extraction process, while the continuous array structure is important for the scale up of the devices for future applications. A typical organic photosynaptic device is composed of a gate electrode (G), divinyltetramethyldisiloxane-bis(benzocyclobutene)

(BCB) and $\mathrm{SiO}_{2}$ insulator layers, Dif-TES-ADT crystal arrays, and source (S) and drain (D) electrodes (Fig. 2a). The $\mathrm{SiO}_{2} / \mathrm{Si}$ substrate was covered with $\mathrm{BCB}$ to minimize traps and guarantee good wettability for organic solvents. As shown in Fig. 2b, a light pulse can be regarded as a presynaptic spike or an external stimulus. The upper surface of the Dif-TES-ADT crystal arrays under light irradiation emulates a presynaptic membrane. The organic semiconductor channel layer with $\mathrm{S}$ and D electrodes acts as a postsynaptic dendrite. The holes from the $\mathrm{S}$ are analogous to neurotransmitters and migrate in response to presynaptic spikes, while the holes between the insulator layer and Dif-TES-ADT crystal arrays emulate synaptic clefts. 

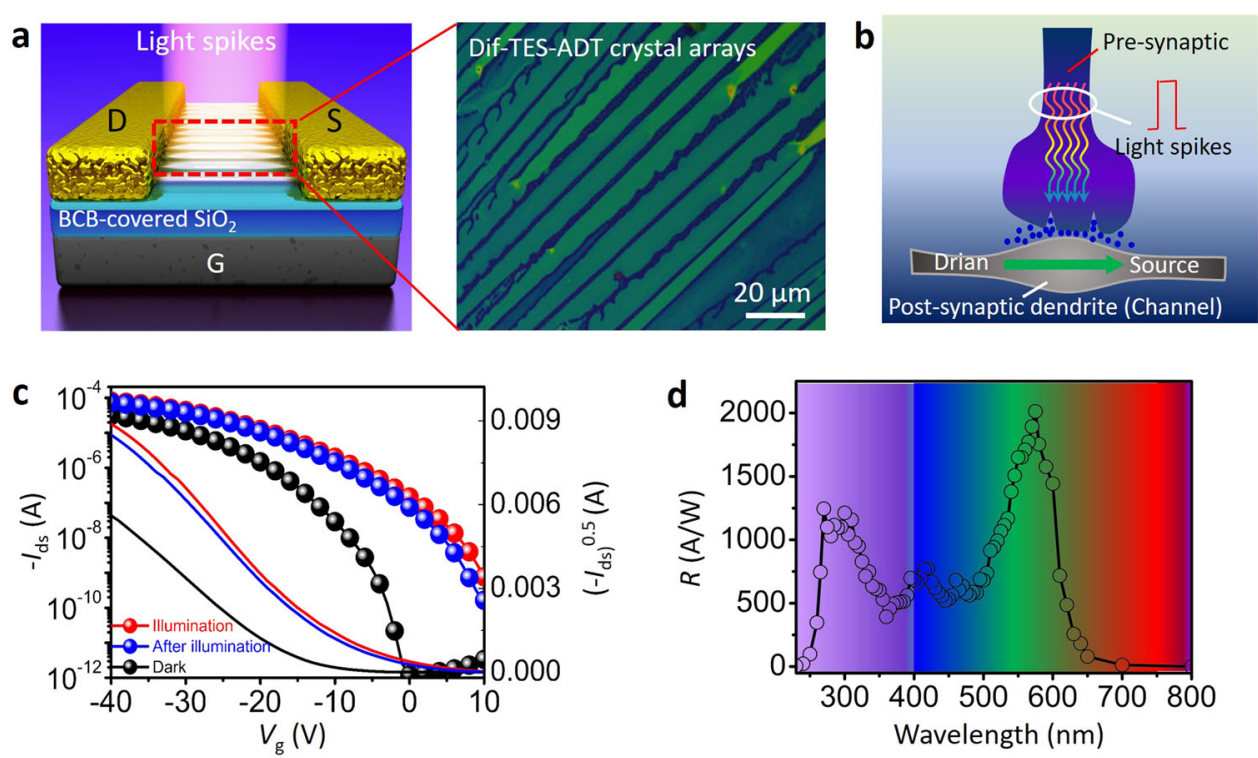

Fig. 2 Performance of the photosynaptic device. a Schematic illustration of the organic photosynaptic device based on an OMC array (left) and cross-polarized optical micrograph of the Dif-TES-ADT crystal array. b Simple schematic of an artificial photosynapse emulating a biological synapse. c Typical transfer characteristics of a photosynaptic device acquired in the dark, under $550 \mathrm{~nm}$ light irradiation $\left(10 \mu \mathrm{W} \mathrm{cm}{ }^{-2}\right)$, and after light irradiation. $\mathbf{d} R$ as a function of light wavelength at a low $V_{\mathrm{g}}$ of $5 \mathrm{~V}$.

First, we investigated the photoresponsive properties of the fabricated organic photosynapses. Figure 2c shows that the device exhibits good p-type transistor characteristics in the dark. When monochromatic light of $575 \mathrm{~nm}$ at $10 \mu \mathrm{W} \mathrm{cm}$ cr $^{-2}$ is vertically irradiated on the device, the S-D current $\left(I_{\mathrm{ds}}\right)$ remarkably increases, along with a positive shift of the threshold voltage $\left(V_{\mathrm{T}}\right)$ from -15.5 to $-8.2 \mathrm{~V}$, indicating easier turn-on of the device under light irradiation. The photoresponsivity $(R)$ is an important parameter to evaluate the sensitivity of a photodetector $^{24-27}$. It is expressed as:

$$
R=\frac{\left(I_{\mathrm{pc}}-I_{\mathrm{dark}}\right)}{P}
$$

where $I_{\mathrm{pc}}$ is the photocurrent, $I_{\mathrm{dark}}$ is the dark current, $P$ is the power, and $E_{\mathrm{hv}}$ is the energy of the incident photon. The $R$ versus gate voltage $\left(V_{\mathrm{g}}\right)$ curve of our device is displayed in Supplementary Fig. S3. The OMC-based photosynaptic device has a higher $R\left(1650 \mathrm{~A} \mathrm{~W}^{-1}\right)$ and a larger $I_{\mathrm{pc}} / I_{\text {dark }}$ ratio $\left(\sim 10^{4}\right)$ at a low $V_{\mathrm{g}}$ of $5 \mathrm{~V}$ compared to the polycrystalline thin-film-based device (Supplementary Fig. S4). This indicates the extremely high light sensitivity of the OMC-based device. The wavelength-dependent $R$ in Fig. $2 \mathrm{~d}$ shows that the photosynaptic device exhibits a broad photoresponse in the visible range and thereby allows recognition of visible signals for emulation of the human vision system. In addition to the high photosensitivity, the device also exhibits a strong persistent photoconductivity behavior, that is, the photocurrent can be retained and slowly decays even after removing the light (Fig. 2c and Supplementary Fig. S5). Interestingly, this slow relaxation behavior is quite similar to the decay process of action potentials that transmit through neurons and can thus be applied in emulating the working principles of photosynapses.

Synapses allow a neuron to pass a signal to another cell. Triggering of the excitatory postsynaptic current (EPSC) is thought to be an important process in assessing neuronal transmission ${ }^{28}$. In our photosynaptic device, a white light spike with a relatively weak light intensity of $5 \mu \mathrm{W}$ $\mathrm{cm}^{-2}$ and a pulse width (W) of $120 \mathrm{~ms}$ was applied to trigger a postsynaptic current at a $V_{\mathrm{ds}}$ of $-30 \mathrm{~V}$ and a $V_{\mathrm{g}}$ of $5 \mathrm{~V}$. Figure $3 \mathrm{a}$ shows that the EPSC rapidly reaches a peak value of $46 \mathrm{pA}$ and then gradually decays back to the initial value of $\sim 6.8 \mathrm{pA}$ within $5 \mathrm{~s}$. This trend emulates the transmission process of an optical signal in a biological manner, in which the incoming light generates an action spike and is transmitted across the photosynaptic device to the next photosynaptic device. At a constant light spike amplitude, the EPSC of the photosynaptic device increases almost linearly from 12 to $62 \mathrm{pA}$ as the spike duration increases from 30 to $180 \mathrm{~ms}$ (Fig. 3b and Supplementary Fig. S6). We note that the EPSC rise for the photosynaptic device is very steep, while the EPSC fall is relatively slow. The rise time $\left(t_{\mathrm{r}}\right)$ and fall time $\left(t_{\mathrm{f}}\right)$ are estimated to be 0.8 and $4.8 \mathrm{~s}$, respectively. This behavior is related to the different accumulation and release rates of photogenerated carriers. It is known that ambient oxygen can diffuse into $\pi$-conjugated OMCs, leading to the formation of deep acceptor levels in the bandgap ${ }^{29}$. The oxygen- 

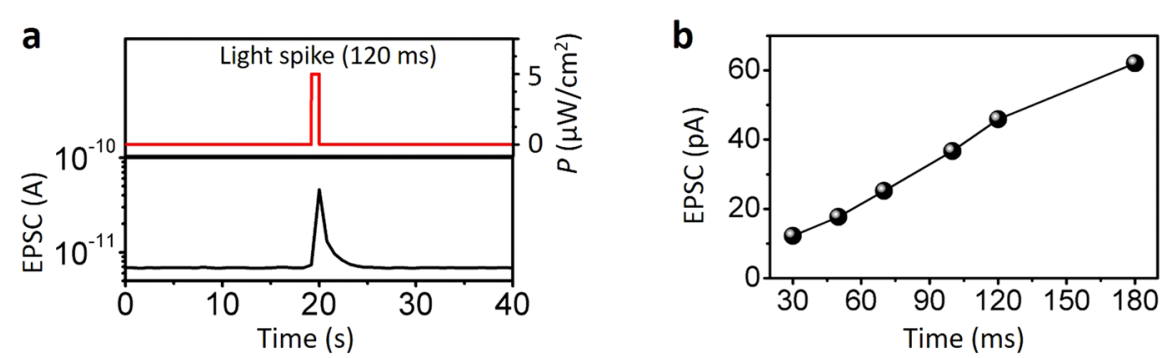

C
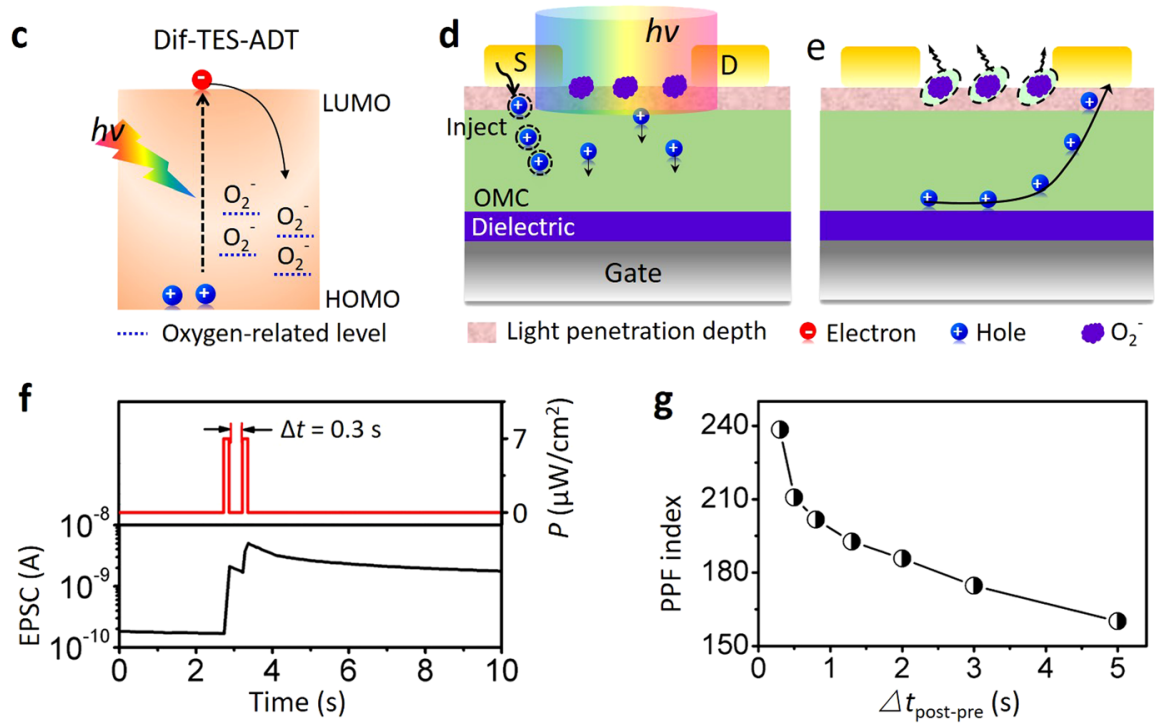

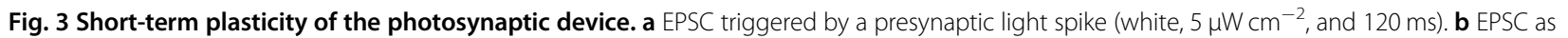
a function of the light pulse width. The white light spike was fixed at $5 \mu \mathrm{W} \mathrm{cm}{ }^{-2} . \mathbf{c}$ Energy diagram showing the photoinduced charge transfer from the $\mathrm{OMC}$ to the oxygen-related level. $\mathbf{d}$, e Schematic illustrations of the working principles of the organic photosynaptic device under weak light stimulation. $\mathbf{f}$ Postsynaptic current triggered by a pair of presynaptic light spikes. $\mathbf{g}$ PPF index as a function of $\Delta t_{\text {post-pre. }}$

related levels could be directly detected using photoemission and absorption spectra ${ }^{30}$ and were found to be $\sim 0.28 \mathrm{eV}$ above the highest occupied molecular orbital for pentacene $^{31}$. To assess the impact of $\mathrm{O}_{2}$ incorporation, we performed a density of states calculation of the energy band structure of the Dif-TES-ADT crystal and indeed revealed the induction of a distinct energy level at $1.8 \mathrm{eV}$ above the Fermi level (Supplementary Fig. S7).

When the device is under light irradiation, electrons and holes will be generated in the light-absorbing Dif-TESADT crystals. Based on the energy band diagram in Fig. 3c, the electrons quickly transfer from Dif-TES-ADT to oxygen-related levels, forming $\mathrm{O}_{2}{ }^{-}$anions, while the holes remain within the Dif-TES-ADT crystals and migrate to the $\mathrm{BCB} / \mathrm{Dif}-\mathrm{TES}-\mathrm{ADT}$ crystal interface. Meanwhile, the $\mathrm{O}_{2}{ }^{-}$anions form a negative space-charge layer on the top surface of Dif-TES-ADT crystals. The negative charges generate a gating effect, which can continuously trigger hole injection from the $\mathrm{S}$ electrode to maintain charge conservation in the device channel (Fig. 3d $)^{32,33}$. Thus the hole concentration in the device rapidly increases, resulting in a fast EPSC rise. Since the penetration depth of light in the Dif-TES-ADT crystals is only $1-5 \mathrm{~nm}$, the formed $\mathrm{O}_{2}{ }^{-}$anions are predominately located on the crystal surfaces under weak light irradiation. After removing the light spike, the superficial $\mathrm{O}_{2}{ }^{-}$ anions are unstable and will gradually vanish (Fig. 3e). This, of course, causes the slow disappearance of the formed negative space-charge layer, leading to gradually weakening of the gating effect. As a result, the hole injection is interrupted, and the EPSC gradual declines, leading to a long $t_{\mathrm{f}}$. The $\mathrm{O}_{2}{ }^{-}$anion release rate can be defined by the following equation:

$$
\text { Rate }=\frac{\mathrm{d} Q}{\mathrm{~d} t}=C \frac{\mathrm{d} V_{T}(t)}{\mathrm{d} t}=-\left\lceil\frac{2 L C}{\mu_{\mathrm{sat}} W}\right\rceil^{0.5} \frac{\mathrm{d}\left(I_{\mathrm{ds}}(t)\right)^{0.5}}{\mathrm{~d} t}
$$

where $C$ is the insulator capacitance per unit area, $W$ is the channel width, $L$ is the channel length, and $\mu_{\text {sat }}$ is the saturation regime mobility. According to the equation, the release rate of the $\mathrm{O}_{2}{ }^{-}$anions is estimated to be $\sim 2.5 \times$ 

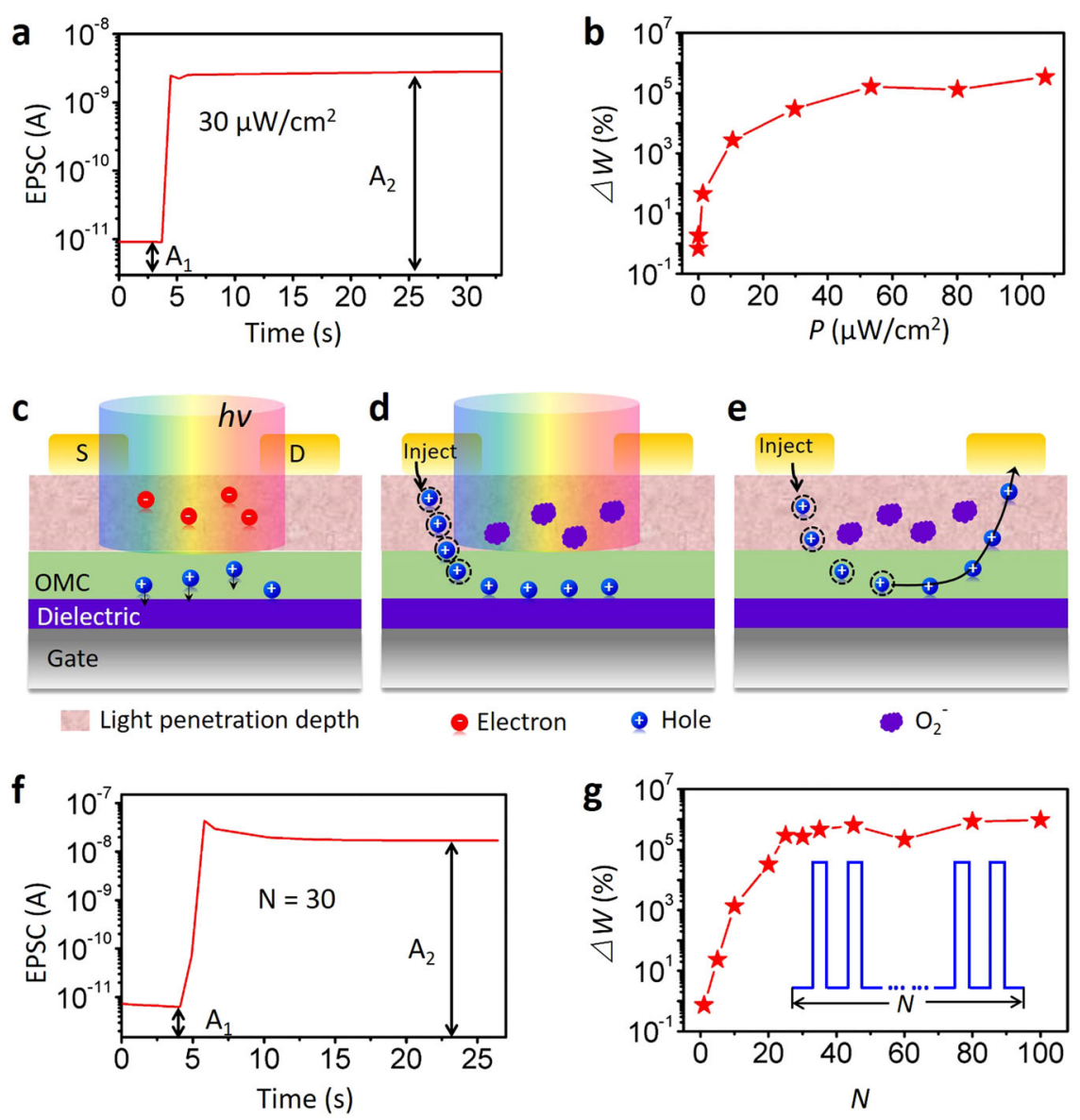

Fig. 4 Long-term plasticity of the photosynaptic device. a EPSC of the organic photosynaptic device for a presynaptic light spike input (white, $30 \mu \mathrm{W} \mathrm{cm}{ }^{-2}$, and $\left.50 \mathrm{~ms}\right)$. b Synaptic $\Delta W$ as a function of the white light spike intensity. The light spike width was fixed at $50 \mathrm{~ms}$. c-e Schematic illustrations of the working principles of the organic photosynaptic device under strong light stimulation. $\mathbf{f}$ EPSC of the organic photosynaptic device for 30 white light spikes with a narrow pulse width of $\sim 20 \mathrm{~ms}$ and a high frequency of $\sim 50 \mathrm{~Hz}$. $\mathbf{g} \Delta W$ as a function of the number of input light pulses.

$10^{9} \mathrm{~cm}^{-2} \mathrm{~s}^{-1}$. To further prove the validity of photoinduced charge transfer from OMCs to oxygen molecules, we compared the transfer characteristics of the device under three different conditions: air, vacuum, and an oxygen atmosphere in the dark (Supplementary Fig. S8). No significant change was observed for the transfer characteristics. However, the photocurrent under a light spike in vacuum is much smaller than that measured in air (Supplementary Fig. S9). These results unambiguously demonstrate the important role of oxygen adsorption in controlling the EPSC in OMCs.

Short-term plasticity (STP) is an important foundation of learning and memory and is believed to be the origin of the short-term memory of the brain ${ }^{34}$. STP refers to the change in the strength of a synapse's response to an external stimulus over a time range of milliseconds to a few minutes. In biological synapses, paired pulse facilitation (PPF) is a fundamental form of STP, in which the EPSC triggered by the spike increases when a second spike closely follows a prior spike. STP is successfully mimicked in our photosynaptic device, as shown by applying two successive light spikes $\left(7 \mu \mathrm{W} \mathrm{cm}^{-2}, 120 \mathrm{~ms}\right)$ with different interspike intervals $\left(\Delta t_{\mathrm{pre}}\right)$. Figure $3 \mathrm{f}$ exhibits the EPSC of the photosynaptic device for a $\Delta t_{\text {pre }}$ of $300 \mathrm{~ms}$. The peak value of the second EPSC spike $\left(\mathrm{A}_{2}\right)$ is 2.38 times that of the first EPSC spike $\left(\mathrm{A}_{1}\right)$. The PPF index is defined by $A_{2} / A_{1} \times 100 \%$, the peak value ratio between the second EPSC $\left(\mathrm{A}_{2}\right)$ and the first $\operatorname{EPSC}\left(\mathrm{A}_{1}\right)$. The PPF

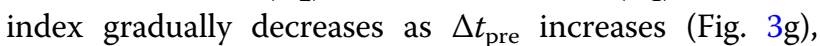
which is similar to the neural response. For a smaller $\Delta t_{\text {pre }}$, this interval is shorter than the release rate of $\mathrm{O}_{2}{ }^{-}$ anions, which thus adds to the total amount of photogenerated holes in the channel during the second-lightspike period. As a result, the second EPSC is higher than the first EPSC.

Long-term plasticity (LTP) is widely regarded as the mechanism of human memory ${ }^{35}$. The synaptic strength will show a persistent increase following a strong external 

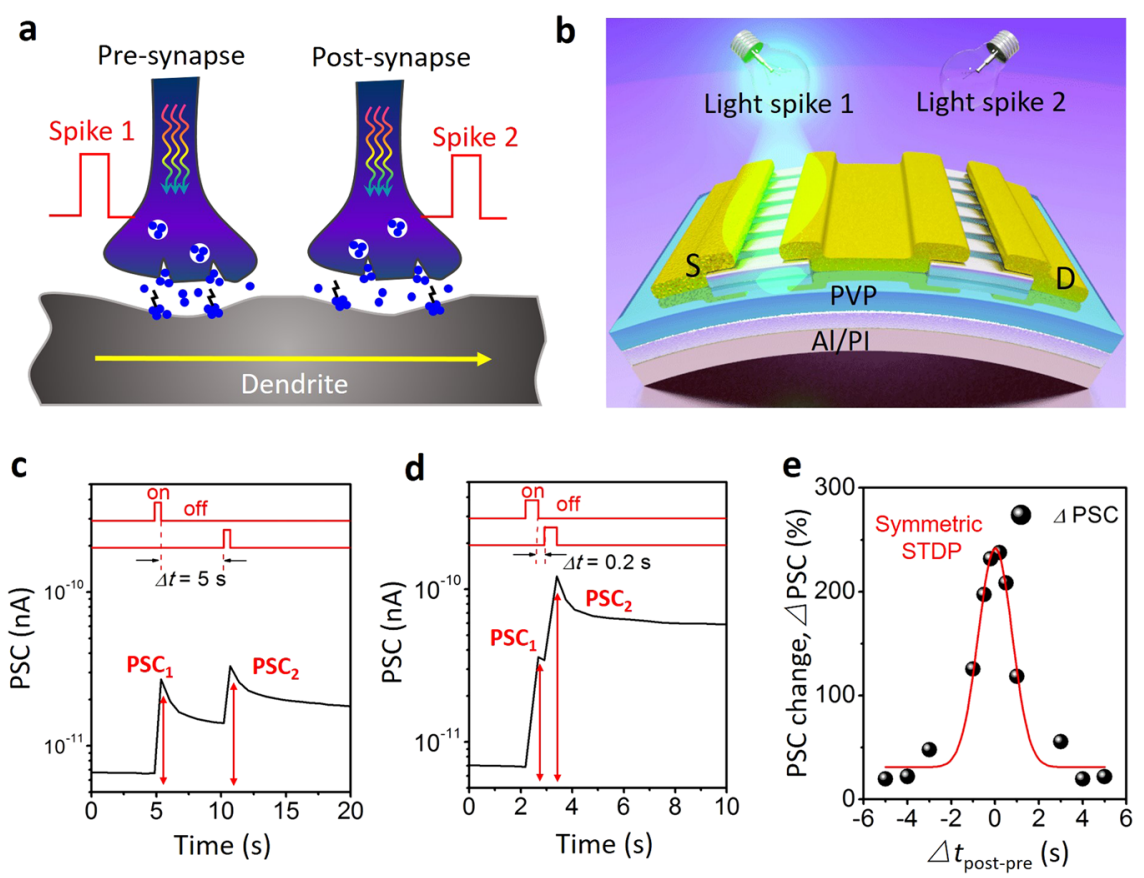

Fig. 5 Spike-timing-dependent plasticity of the photosynaptic device. a Schematic image of photosynaptic integration. b Schematic showing two connected photosynaptic devices for the emulation of spike-timing-dependent plasticity. $\mathbf{c}, \mathbf{d}$ PSC variation for different $\Delta t$ values: $\mathbf{c} \Delta t=+5 \mathrm{~s}$ and $\mathbf{d} \Delta t=+0.2 \mathrm{~s}$. e PSC change as a function of $\Delta t_{\text {post-pre. }}$

stimulation or a number of successive stimulations. In analogy to biological synapses, LTP in the photosynaptic device is represented by the phenomenon in which the EPSC of the device can persist for a long time after removing light stimulation. To assess the LTP of the photosynapse, a stronger light spike (white, $30 \mu \mathrm{W} \mathrm{cm}{ }^{-2}$, and $50 \mathrm{~ms}$ ) was input into the Dif-TES-ADT crystals to obtain the EPSC. Figure 4a shows the EPSC retention curve after the light spike. A nonvolatile increase from the initial state of $A_{1}(9.2 \mathrm{pA})$ to the final state of $A_{2}(2.7 \mathrm{nA})$ was observed. After removing the external light stimuli, it took approximately $190 \mathrm{~s}$ for the device to return to the initial state (Supplementary Fig. S10). In addition, the synaptic weight change, $\Delta W\left(\left(\mathrm{~A}_{2}-\mathrm{A}_{1}\right) / \mathrm{A}_{1} \times 100 \%\right)$, is $3.0 \times 10^{4} \%$ in the Dif-TES-ADT crystal-based device. In contrast, when the polycrystalline Dif-TES-ADT thin film was used as the active layer, the EPSC showed a more rapid drop after light stimulation, and $\Delta W(128 \%)$ was significantly reduced owing to the existence of many structural defects (e.g., misorientations, voids, and grain boundaries) in the thin film (Supplementary Fig. S11). For the Dif-TES-ADT crystal-based device, $\Delta W$ as a function of the light intensity is depicted in Fig. 4b. As the intensity of the light pulse increases from 0.001 to $53 \mu \mathrm{W} \mathrm{cm}{ }^{-2}$, $\Delta W$ linearly increases from $\sim 0.4$ to $1.6 \times 10^{5} \%$. However, when the intensity of the light pulse is $>55 \mu \mathrm{W} \mathrm{cm} \mathrm{cm}^{-2}, \Delta \mathrm{W}$ starts to gradually saturate. These results indicate that our artificial photosynapses show a better memory effect when facing a more impressive activity event. $\Delta W$ can also be well modulated by $V_{\mathrm{g}}$, as shown in Supplementary Fig. S12. This means that the synaptic plasticity is modulated by both electrical and light stimuli, which can mimic dopamine-facilitated synaptic activity ${ }^{36}$. The mechanism of LTP mimicry, in this case, can be explained as follows: a stronger light stimulus can generate more carriers in DifTES-ADT crystals, thus increasing the concentration of $\mathrm{O}_{2}{ }^{-}$anions (Fig. 4c, d). In this scenario, the highconcentration $\mathrm{O}_{2}{ }^{-}$anions undergo long-range diffusion, leading to the formation of oxygen-related traps inside the crystals. As a result, the lifetime and stability of the $\mathrm{O}_{2}{ }^{-}$ anions are considerably increased compared to those of $\mathrm{O}_{2}{ }^{-}$anions produced under weak light. Therefore, even after stopping the light spike, the $\mathrm{O}_{2}{ }^{-}$anions can remain in the Dif-TES-ADT crystals for a relatively long time and consequently maintain the device in a highly conductive state over a long retention time (Fig. 4e). This feature makes OMCs a unique platform for emulating the LTP process in photosynapses. In addition to a stronger light spike, a higher number $(N)$ of weak light stimulations with a short interval can also enhance the connection strength between the photosynapses. Figure $4 \mathrm{f}$ shows the EPSC- $t$ curve for the aforementioned stimulation protocol with 30 weak light spikes $\left(5 \mu \mathrm{W} \mathrm{cm}{ }^{-2}\right.$, a narrow pulse width of $\sim 20 \mathrm{~ms}$, and a high frequency of $\sim 50 \mathrm{~Hz}$ ). An obvious enhancement in the EPSC was observed after 30 light spikes, with a total $\Delta W$ enhancement of $\sim 9.5 \times 10^{5} \%$. 
a
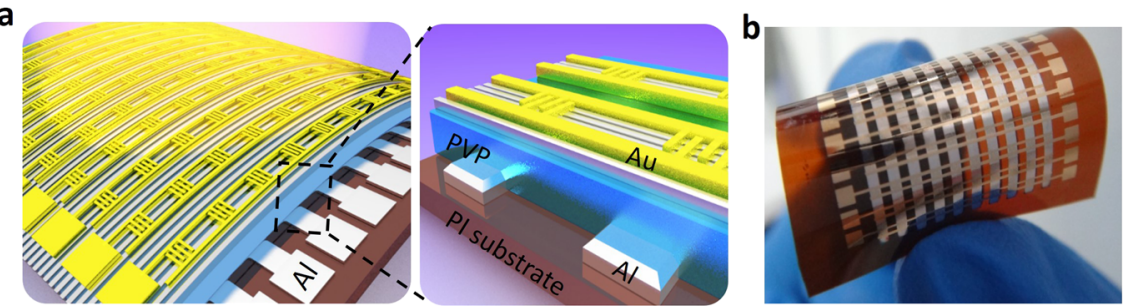

C
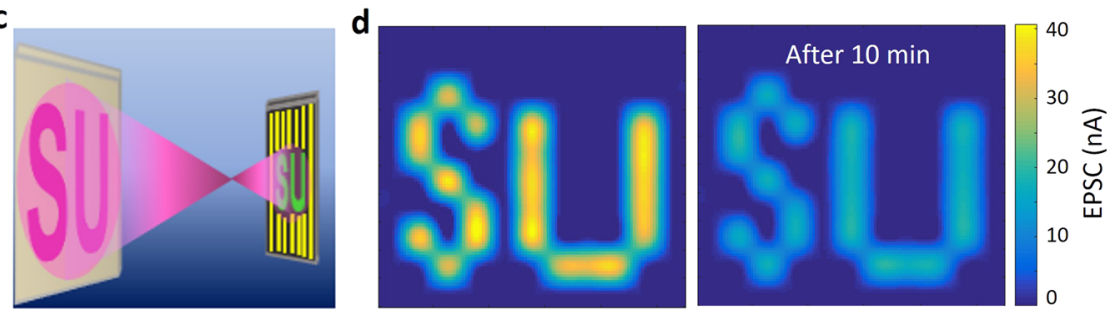

Fig. 6 Flexible visual-perception system. a Schematic illustration and $\mathbf{b}$ photograph of the photosynaptic device array on a flexible PI substrate c Original and imaged letter "SU" and $\mathbf{d}$ EPSC mappings for the letters "SU" immediately (left) or 10 min after light exposure.

Note that the EPSC can persist for hours after removing the light irradiation (Supplementary Fig. S13). Only when a large negative gate bias of $-40 \mathrm{~V}$ is applied does the EPSC quickly return to a low conductivity state, realizing a fast restorability of the synaptic weight (Supplementary Fig. S14). In addition, $\Delta W$ increases as the $N$ of the presynaptic stimuli increases (Fig. 4g), suggesting that all stimulation signals are accepted by the photosynapse. These results demonstrate that many transient light signals can be transferred into long-term memory via a consolidation process in the photosynaptic devices, which is consistent with the psychological model of human memory (Supplementary Fig. S15).

In biological synapses, STDP, known as the Hebbian learning rule, is an essential function ${ }^{14}$. It refers to the magnitude of the connection strength, which closely depends on the relative timing of prespike and postspike activities. Figure 5a schematically illustrates the photosynaptic integration. To emulate the STDP function, two organic photosynaptic devices were integrated by connecting them with a common Au electrode (Fig. 5b). To avoid light spike 1 illuminating the other photosynaptic device, we placed an aluminum foil at the middle of the two devices, as illustrated in Supplementary Fig. S16. One photosynaptic device is regarded as a presynapse, while the other one is regarded as a postsynapse. Two synaptic light spikes (white, $8 \mu \mathrm{W} \mathrm{cm} \mathrm{cm}^{-2}$, and $500 \mathrm{~ms}$ ) were separately applied to the presynapse and postsynapse, with a varied time interval $(\Delta t)$. In this case, the connection strength between the two photosynaptic devices is defined as $\triangle \mathrm{PSC}=\left(\mathrm{PSC}_{2}-\mathrm{PSC}_{1}\right) / \mathrm{PSC}_{1}$, where $\mathrm{PSC}$ represents the postsynaptic current. Figure $5 \mathrm{c}, \mathrm{d}$ show the variation in the PSC when $\Delta t$ is +5 and $+0.2 \mathrm{~s}$. As $\Delta t$ increases, the connection strength is weakened. A typical symmetric form of STDP induced by temporal correlations of the presynaptic and postsynaptic spikes is obtained (Fig. 5e). The symmetric STDP characteristic is important for emulating more complicated neuromorphic functions of the brain.

To simulate the visual-perception processes of humans, we further integrated photosynaptic devices on a flexible polyimide (PI) substrate (Fig. 6a). An active-matrix array with $10 \times 10$ photosynapses was constructed on a semitransparent, flexible substrate $\left(2 \times 2 \mathrm{~cm}^{2}\right)$ (Fig. 6b). Supplementary Fig. S17 shows a top-view optical microscopic image of the photosynaptic device array. Next, the image recognition and memory capabilities of the photosynapse array were tested. We first imaged the characters "SU" on the device array by irradiating the devices above the "SU" characters with white light $\left(150 \mu \mathrm{W} \mathrm{cm}{ }^{-2}\right.$; Fig. 6c). It is noteworthy that these characters can be clearly resolved (Fig. 6d), revealing the reliable imaging function of the device. In addition, after removing the optical signal, the "SU" characters can still be clearly read from the activematrix array at $10 \mathrm{~min}$. This verifies that the visualmemory function of human beings has been successfully mimicked. Note that the EPSC for the photosynapses under or after light exposure is distributed in a relatively narrow range with little fluctuation, confirming the high uniformity and stability of the visual-perception system (Supplementary Fig. S18). The mechanical flexibility of the OMC-based photosynaptic device was further investigated. The synaptic performances under different bending radii (from a flat state to $7.0 \mathrm{~mm}$ ) were recorded in situ, as shown in Supplementary Fig. 19a. $\Delta W$ decreases slightly with decreasing bending radius (Supplementary Fig. 19b). Furthermore, the photosynaptic performance of the flexible device was tested before and after multiple 
bending stresses were applied. At a small bending radius of $7.0 \mathrm{~mm}$, the $\Delta W$ of the device slightly changes even after 2500 bending cycles (Supplementary Fig. 20). This result shows that the photosynaptic devices have outstanding flexibility and are very robust against bending strain, suggesting the great potential of the OMC-based photosynaptic devices in next-generation flexible electronics.

\section{Conclusions}

In conclusion, we successfully demonstrate a new concept of an organic photosynaptic device that features synaptic and optical-sensing functions in a single device, which successfully avoids the use of sophisticated device architectures. By taking advantage of the high photosensitivity of OMCs and the unique photon-induced charge transfer effect, the photosynaptic device is capable of directly transmitting, memorizing, recognizing, and learning a light stimulus in a manner analogous to the biological neural system. Therefore, various important biological visual-perception functions, including STP and LTP behaviors and STDP characteristics, are successfully emulated. Furthermore, as a proof of concept, an artificial visual-perception system with $10 \times 10$ photosynapse pixels was constructed on a flexible substrate, which shows remarkable capabilities for recognizing and memorizing optical images. This artificial visual-perception system has promise for application in future visual prosthetics and intelligent products.

\section{Materials and methods Preparation and characterizations}

A solution of $3 \mathrm{mg} \mathrm{mL}^{-1}$ Dif-TES-ADT (obtained from Luminescence Technology Corp) was prepared in toluene (99.9\%, Sigma-Aldrich). Afterwards, $\sim 10 \mu \mathrm{L}$ of Dif-TESADT solution was injected between a $\mathrm{BCB}$-covered $\mathrm{SiO}_{2} /$ Si substrate (typically a $1.5 \times 1.5 \mathrm{~cm}^{2}$ silicon wafer with a $300-\mathrm{nm}$ thermally grown oxide) and a blade. The electrochemically active group-free $\mathrm{BCB}$ insulator layer $(\sim 50 \mathrm{~nm})$ was used to passivate the - $\mathrm{OH}$ groups on the $\mathrm{SiO}_{2}$ surface and avoid electron trapping on the dielectric. As the blade was withdrawn at a constant speed of $110 \mu \mathrm{m} \mathrm{s}^{-1}$, the Dif-TES-ADT solution evaporated, forming crystal arrays on the substrate (Supplementary Fig. S1a). After the fabrication of Dif-TES-ADT crystal arrays, a cross-polarized optical microscope was used to verify the macroscopic molecular ordering and the absence of polycrystalline domains across the Dif-TESADT single crystals. Supplementary Fig. S1b, c shows uniform brightness across the entire crystal, with the intensity of the brightness depending on the angle between the long axis of the crystal and the polarization plane of the incident light. Polar plots of the average brightness over three distinct regions reveal identical birefringence (Supplementary Fig. S1d), which is known to occur only in a structurally pristine single crystal.

\section{Device fabrication and measurement}

Organic photosynaptic devices were made on BCBcovered $\mathrm{SiO}_{2} / \mathrm{Si}$ substrates. An $\mathrm{Au}(50 \mathrm{~nm})$ layer was deposited through a metal mask to form S/D electrodes. All devices were measured using a semiconductor parameter analyzer (Keithley 4200-SCS). Light pulses were generated using a function generator. Monochromatic light in the ultraviolet-visible range was captured by a spectrometer with optical filters, and a power meter was used to determine the light intensity.

\section{Photosynapse array fabrication and characterization}

PI of $120-\mu \mathrm{m}$ thickness (DuPont ${ }^{\mathrm{TM}}$ Kapton $^{\circledR}$ HPP-ST) was utilized as the substrate, and it was mounted on a $\mathrm{Si}$ substrate to maintain a flat state for ease of handling during fabrication. Then patterned gate electrodes (Ag, $100 \mathrm{~nm}$ ) were thermally evaporated at $0.4 \mathrm{~nm} \mathrm{~s}^{-1}$ under vacuum. A crosslinked poly(4-vinylphenol) (PVP) dielectric was prepared by spin-coating a solution containing $11 \mathrm{wt} \%$ PVP and $4.7 \mathrm{wt} \%$ poly(melamine-co-formaldehyde) methylated in propylene glycol monomethyl ether acetate solvent on the substrate at $2500 \mathrm{rpm}$ for $20 \mathrm{~s}$. After that, the substrate was annealed at $160^{\circ} \mathrm{C}$ for $2 \mathrm{~h}$ in a nitrogen atmosphere. Next, Dif-TES-ADT crystal arrays were fabricated by blade coating. The blade-coating conditions were as follows: the Dif-TES-ADT solution concentration was $4 \mathrm{mg} \mathrm{mL}^{-1}$, and the blade-coating speed was $150 \mu \mathrm{m} \mathrm{s}^{-1}$. The Dif-TES-ADT crystal arrays were patterned by scratching with a probe tip on an $X Y$ translation stage. Patterned $100 \mathrm{~nm}$ Au S-D electrodes were thermally evaporated through a shadow mask to form a photosynaptic device array. The characters "SU" were projected on the device array by irradiating the devices above the "SU" characters with white light. The currents of each device in the array were measured before irradiation, under white light irradiation $\left(150 \mu \mathrm{W} \mathrm{cm}{ }^{-2}\right.$, $85 \mathrm{~s}$ ), and after removing light using a Keithley 4200-SCS system. Afterward, the currents immediately after light irradiation and $10 \mathrm{~min}$ after removing the light were mapped.

\section{Acknowledgements}

We thank Kaifeng Niu, Youyong Li, and Lifeng Chi for the DOS calculation. This work was supported by the National Natural Science Foundation of China (Nos. 51672180, 51622306, 21673151), the Natural Science Foundation of Jiangsu Province of China (BK20180845), the Collaborative Innovation Center of Suzhou Nano Science and Technology (NANO-CIC), the Priority Academic Program Development of Jiangsu Higher Education Institutions (PAPD), the Qing Lan Project, the 111 project, and the Joint International Research

Laboratory of Carbon-Based Functional Materials and Devices.

\section{Conflict of interest}

The authors declare that they have no conflict of interest. 


\section{Publisher's note}

Springer Nature remains neutral with regard to jurisdictional claims in published maps and institutional affiliations.

Supplementary information is available for this paper at https://doi.org/ 10.1038/s41427-019-0182-2.

Received: 11 July 2019 Revised: 15 September 2019 Accepted: 3 October 2019.

Published online: 13 December 2019

\section{References}

1. Hillis, J. M., Ernst, M. O., Banks, M. S. \& Landy, M. S. Combining sensory information: mandatory fusion within, but not between, senses. Science $\mathbf{2 9 8}$ 1627-1630 (2002)

2. Ko, H. C. et al. A hemispherical electronic eye camera based on compressible silicon optoelectronics. Nature 454, 748-752 (2008).

3. Wang, Z. R. et al. Fully memristive neural networks for pattern classification with unsupervised learning. Nat. Electron. 1, 137-145 (2018).

4. Mossman, K. \& Groves, J. Micropatterned supported membranes as tools for quantitative studies of the immunological synapse. Chem. Soc. Rev. 36, 46-54 (2007).

5. Wang, K. et al. Light-stimulated synaptic transistors fabricated by a facile solution process based on inorganic perovskite quantum dots and organic semiconductors. Small 15, 1900010 (2019).

6. Zhai, Y. B. et al. Toward non-volatile photonic memory: concept, material and design. Mater. Horiz. 5, 641-654 (2018).

7. He, Y. L., Yang, Y., Nie, S., Liu, R. \& Wan, Q. Electric-double-layer transistors for synaptic devices and neuromorphic systems. J. Mater. Chem. C 6, 5336-5352 (2018).

8. Zhu, B., Liu, J. Z., Cauley, S. F., Rosen, B. R. \& Rosen, M. S. Image reconstruction by domain-transform manifold learning. Nature 555, 487-492 (2018).

9. Benfenati, $\mathrm{V}$. et al. A transparent organic transistor structure for bidirectional stimulation and recording of primary neurons. Nat. Mater. 12, 672-680 (2013).

10. Zang, Y. P., Shen, H. G., Huang, D. Z., Di, C. A. \& Zhu, D. B. A dual-organictransistor-based tactile-perception system with signal-processing functionality. Adv. Mater. 29, 1606088 (2017).

11. Muccini, M. A bright future for organic field-effect transistors. Nat. Mater. 5, 605-613 (2006).

12. Wang, H. L. et al. Ferroelectric/electrochemical modulated organic synapse for ultraflexible, artifcial visual-perception system. Adv. Mater. 30, 1803961 (2018).

13. Lee, Y. et al. Stretchable organic optoelectronic sensorimotor synapse. Sci. Adv. 4, eaat7387 (2018).

14. Wang, Y. et al. Photonic synapses based on inorganic perovskite quantum dots for neuromorphic computing. Adv. Mater. 30, 1802883 (2018).

15. Dai, S. et al. Light-stimulated synaptic devices utilizing interfacial effect of organic field-effect transistors. ACS Appl. Mater. Interfaces 10, 21472-21480 (2018).

16. Tsurumi, J. et al. Coexistence of ultra-long spin relaxation time and coherent charge transport in organic single-crystal semiconductors. Nat. Phys. 13, 994-999 (2017).
17. Deng, W. et al. Channel-restricted meniscus self-assembly for uniformly aligned growth of single-crystal arrays of organic semiconductors. Mater. Today 24, 17-25 (2019).

18. Deng, W. et al. Wafer-scale precise patterning of organic single-crystal nanowire arrays via a photolithography-assisted spin-coating method. Adv. Mater. 27, 7305-7312 (2015).

19. Jiang, $H$. et al. Hole mobility modulation in single-crystal metal phthalocya-

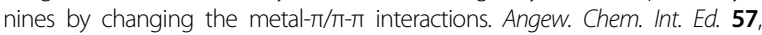
10112-10274 (2018).

20. Kim, J. et al. Ultrahigh detective heterogeneous photosensor arrays with inpixel signal boosting capability for large-area and skin-compatible electronics. Adv. Mater. 28, 3078-3086 (2016).

21. Jurchescu, O. D. et al. Organic single-crystal field-effect transistors of a soluble anthradithiophene. Chem. Mater. 20, 6733-6737 (2008).

22. Niazi, M. R. et al. Solution-printed organic semiconductor blends exhibiting transport properties on par with single crystals. Nat. Commun. 6, 8598 (2015).

23. Gundlach, D. J. et al. Contact-induced crystallinity for high-performance soluble acene-based transistors and circuits. Nat. Mater. 7, 216-221 (2008).

24. Deng, W. et al. Aligned single-crystalline perovskite microwire arrays for highperformance flexible image sensors with long-term stability. Adv. Mater. 28, 2201-2208 (2016).

25. Deng, W. et al. Ultrahigh-responsivity photodetectors from perovskite nanowire arrays for sequentially tunable spectral measurement. Nano Lett. 17, 2482-2489 (2017).

26. Zhang, X. J. et al. Alignment and patterning of ordered small-molecule organic semiconductor micro-nanocrystals for device applications. Adv. Mater. 28 2475-2530 (2016).

27. Xu, X. Z. et al. Saturated vapor-assisted growth of single-crystalline organicinorganic hybrid perovskite nanowires for high-performance photodetectors with robust stability. ACS Appl. Mater. Interfaces 10, 10287-10295 (2018).

28. Xu, W. T., Min, S. Y., Hwang, H. S. \& Lee, T. W. Organic core-sheath nanowire artificial synapses with femtojoule energy consumption. Sci. Adv. 2, e1501326 (2016).

29. Najafov, H., Mastrogiovanni, D., Garfunkel, E., Feldman, L. C. \& Podzorov, V. Photon-assisted oxygen diffusion and oxygen-related traps in organic semiconductors. Adv. Mater. 23, 981-985 (2011).

30. Mitrofanov, O. et al. Oxygen-related band gap state in single crystal rubrene Phys. Rev. Lett. 97, 166601 (2006).

31. Krellner, $C$. et al. Density of bulk trap states in organic semiconductor crystals: Discrete levels induced by oxygen in rubrene. Phys. Rev. B 75, 245115 (2007).

32. Konstantatos, G. et al. Hybrid graphene-quantum dot phototransistors with ultrahigh gain. Nat. Nanotechnol. 7, 363-368 (2012).

33. Ogawa, S. et al. Photoinduced doping effect of pentacene field effect transistor in oxygen atmosphere studied by displacement current measurement. Appl. Phys. Lett. 86, 252104 (2005).

34. Xiao, Z. G. \& Huang, J. S. Energy-efficient hybrid perovskite memristors and synaptic devices. Adv. Electron. Mater. 2, 1600100 (2016).

35. Ohno, T. et al. Short-term plasticity and long-term potentiation mimicked in single inorganic synapses. Nat. Mater. 10, 591-595 (2011).

36. Ham, S., Choi, S., Cho, H., Na, S.-I. \& Wang, G. Photonic organolead halide perovskite artifcial synapse capable of accelerated learning at low power inspired by dopamine-facilitated synaptic activity. Adv. Funct. Mater. 29, 1806646 (2019). 